\title{
The Cryogenic Underground Observatory for Rare Events: Status and Prospects
}

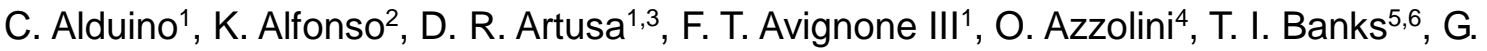
Bari $^{7}$, J.W. Beeman ${ }^{8}$, F. Bellini ${ }^{9,10}$, A. Bersani ${ }^{11}$, M. Biassoni ${ }^{12,13}$, A. Branca ${ }^{14}$, C. Brofferio $^{12,13}$, C. Bucci ${ }^{3}$, A. Camacho ${ }^{4}$, A. Caminata ${ }^{11}$, L. Canonica ${ }^{15,3}$, X. G. Cao ${ }^{16}, \mathrm{~S}$. Capelli $^{12,13}$, L. Cappelli ${ }^{3}$, L. Carbone ${ }^{13}$, L. Cardani ${ }^{9,10}$,a, P. Carniti ${ }^{12,13}$, N. Casali ${ }^{9,10}$, L. Cassina $^{12,13}$, D. Chiesa ${ }^{12,13}$, N. Chott ${ }^{1}$, M. Clemenza ${ }^{12,13}$, S. Copello ${ }^{17,11}$, C. Cosmelli, ${ }^{9,10}$ O. Cremonesi ${ }^{13}$, R. J. Creswick ${ }^{1}$, J. S. Cushman ${ }^{18}$, A. D'Addabbo ${ }^{3}$, I. Dafinei ${ }^{10}$, C. J. Davis $^{18}$, S. Dell'Oro ${ }^{3,19}$, M. M. Deninno ${ }^{7}$, S. Di Domizio ${ }^{17,11}$, M. L. Di Vacri3,20, A. Drobizhev $^{5,6}$, D. Q. Fang ${ }^{16}$, M. Faverzani ${ }^{12,13}$, G. Fernandes ${ }^{17,11}$, E. Ferri ${ }^{13}$, F. Ferroni ${ }^{9,10}$, E. Fiorini ${ }^{13,12}$, M. A. Franceschi ${ }^{21}$, S. J. Freedman ${ }^{6,5, b}$, B. K. Fujikawa ${ }^{6}$, A. Giachero $^{13}$, L. Gironi $^{12,13}$, A. Giuliani' 22 , L. Gladstone ${ }^{15}$, P. Gorla ${ }^{3}$, C. Gotti ${ }^{12,13}$, T. D. Gutierrez ${ }^{23}$, E. E. Haller $^{8,24}$, K. Han ${ }^{25}$, E. Hansen ${ }^{15,2}$, K. M. Heeger ${ }^{18}$, R. Hennings-Yeomans ${ }^{5,6}$, K. P. Hickerson $^{2}$, H. Z. Huang ${ }^{2}$, R. Kadel ${ }^{26}$, G. Keppel ${ }^{4}$, Yu. G. Kolomensky ${ }^{5,26,6}$, A. Leder ${ }^{15}$, C. Ligi ${ }^{21}$, K. E. Lim ${ }^{18}$, Y. G. Ma ${ }^{16}$, M. Maino ${ }^{12,13}$, L. Marini' ${ }^{17,11}$, M. Martinez ${ }^{9,10,27}$, R. H. Maruyama $^{18}$, Y. Mei ${ }^{6}$, N. Moggi ${ }^{28,7}$, S. Morganti ${ }^{10}$, P. J. Mosteiro ${ }^{10}$, T. Napolitano ${ }^{21}$, C. Nones $^{29}$, E. B. Norman ${ }^{30,31^{\star}}$, V. Novati ${ }^{22}$, A. Nucciotti ${ }^{12,13}$, T. O'Donnell ${ }^{32,5,6}$, J. L. Ouellet $^{15}$, C. E. Pagliarone ${ }^{3,33}$, M. Pallavicini ${ }^{17,11}$, V. Palmieri ${ }^{4}$, L. Pattavina ${ }^{3}$, M. Pavan $^{12,13}$, G. Pessina ${ }^{13}$, V. Pettinacci10, G. Piperno ${ }^{9,10, c}$, C. Pira ${ }^{4}$, S. Pirro ${ }^{3}$, S. Pozzi ${ }^{12,13}$, E. Previtali ${ }^{13}$, C. Rosenfeld ${ }^{1}$, C. Rusconi ${ }^{13}$, S. Sangiorgio ${ }^{30}$, D. Santone ${ }^{3,20}$, N. D. Scielzo ${ }^{30}$, V. Singh ${ }^{5}$, M. Sisti ${ }^{12,13}$, A. R. Smith ${ }^{6}$, L. Taffarello ${ }^{14}$, M. Tenconi ${ }^{22,}$ F. Terranova $^{12,13}$, C. Tomei $^{10}$, S. Trentalange ${ }^{2}$, M. Vignati ${ }^{10}$, S. L. Wagaarachchi ${ }^{5,6}$, B. S. Wang $^{30,31}$, H. W. Wang ${ }^{16}$, J. Wilson ${ }^{1}$, L. A. Winslow ${ }^{15}$, T. Wise ${ }^{18,34}$, A. Woodcraft ${ }^{35}$, L. Zanotti $^{12,13}$, G. Q. Zhang ${ }^{16}$, B. X. Zhu' ${ }^{2}$, S. Zimmermann ${ }^{36}$, S. Zucchelli ${ }^{37,7}$

\footnotetext{
${ }^{1}$ Department of Physics and Astronomy, University of South Carolina, Columbia, SC 29208, USA

${ }^{2}$ Department of Physics and Astronomy, University of California, Los Angeles, CA 90095, USA

${ }^{3}$ INFN - Laboratori Nazionali del Gran Sasso, Assergi (L'Aquila) I-67010, Italy

${ }^{4}$ INFN - Laboratori Nazionali di Legnaro, Legnaro (Padova) I-35020, Italy

${ }^{5}$ Department of Physics, University of California, Berkeley, CA 94720, USA

${ }^{6}$ Nuclear Science Division, Lawrence Berkeley National Laboratory, Berkeley, CA 94720, USA

${ }^{7}$ INFN - Sezione di Bologna, Bologna I-40127, Italy

${ }^{8}$ Materials Science Division, Lawrence Berkeley National Laboratory, Berkeley, CA 94720, USA

${ }^{9}$ Dipartimento di Fisica, Sapienza Università di Roma, Roma I-00185, Italy

${ }^{10}$ INFN - Sezione di Roma, Roma I-00185, Italy

${ }^{11}$ INFN - Sezione di Genova, Genova I-16146, Italy

${ }^{12}$ Dipartimento di Fisica, Università di Milano-Bicocca, Milano I-20126, Italy

${ }^{13}$ INFN - Sezione di Milano Bicocca, Milano I-20126, Italy

${ }^{14}$ INFN - Sezione di Padova, Padova I-35131, Italy

${ }^{15}$ Massachusetts Institute of Technology, Cambridge, MA 02139, USA

${ }^{16}$ Shanghai Institute of Applied Physics, Chinese Academy of Sciences, Shanghai 201800, China
} 
${ }^{17}$ Dipartimento di Fisica, Università di Genova, Genova I-16146, Italy

${ }^{18}$ Department of Physics, Yale University, New Haven, CT 06520, USA

${ }^{19}$ INFN - Gran Sasso Science Institute, L'Aquila I-67100, Italy

${ }^{20}$ Dipartimento di Scienze Fisiche e Chimiche, Università dell'Aquila, L'Aquila I-67100, Italy

${ }^{21}$ INFN - Laboratori Nazionali di Frascati, Frascati (Roma) I-00044, Italy

${ }^{22}$ CSNSM, Univ. Paris-Sud, CNRS/IN2P3, Université Paris-Saclay, 91405 Orsay, France

${ }^{23}$ Physics Department, California Polytechnic State University, San Luis Obispo, CA 93407, USA

${ }^{24}$ Department of Materials Science and Engineering, University of California, Berkeley, CA 94720, USA

${ }^{25}$ Department of Physics and Astronomy, Shanghai Jiao Tong University, Shanghai 200240, China

${ }^{26}$ Physics Division, Lawrence Berkeley National Laboratory, Berkeley, CA 94720, USA

${ }^{27}$ Laboratorio de Fisica Nuclear y Astroparticulas, Universidad de Zaragoza, Zaragoza 50009, Spain

${ }^{28}$ Dipartimento di Scienze per la Qualità della Vita, Alma Mater Studiorum - Università di Bologna, Bologna I-47921, Italy

${ }^{29}$ Service de Physique des Particules, CEA / Saclay, 91191 Gif-sur-Yvette, France

${ }^{30}$ Lawrence Livermore National Laboratory, Livermore, CA 94550, USA

${ }^{31}$ Department of Nuclear Engineering, University of California, Berkeley, CA 94720, USA

32 Center for Neutrino Physics, Virginia Polytechnic Institute and State University, Blacksburg, Virginia 24061, USA

${ }^{33}$ Dipartimento di Ingegneria Civile e Meccanica, Università degli Studi di Cassino e del Lazio Meridionale, Cassino I-03043, Italy

${ }^{34}$ Department of Physics, University of Wisconsin, Madison, WI 53706, USA

${ }^{35}$ SUPA, Institute for Astronomy, University of Edinburgh, Blackford Hill, Edinburgh EH9 3HJ, UK

${ }^{36}$ Engineering Division, Lawrence Berkeley National Laboratory, Berkeley, CA 94720, USA

${ }^{37}$ Dipartimento di Fisica e Astronomia, Alma Mater Studiorum - Università di Bologna, Bologna I40127, Italy

a Presently at: Physics Department, Princeton University, Princeton, NJ 08544, USA

${ }^{b}$ Deceased

${ }^{c}$ Presently at: INFN - Laboratori Nazionali di Frascati, Frascati (Roma) I-00044, Italy

* Speaker

E-mail: ebnormanelbl.gov

The Cryogenic Underground Observatory for Rare Events (CUORE) is a large-scale double beta decay experiment utilizing cryogenic bolometers that is currently being commissioned at the Gran Sasso National Laboratory (LNGS) in Italy. Its primary focus is to search for the neutrinoless double beta decay of ${ }^{130} \mathrm{Te}$ with a projected sensitivity to Majorana neutrino masses near the inverted mass hierarchy region. The detector is composed of $9885 \times 5 \times 5-\mathrm{cm}^{3} \mathrm{TeO}_{2}$ crystals of natural isotopic composition arranged in 19 towers of 52 crystals each, all housed in a common dilution refrigerator. A single CUORE-like tower, CUORE-0, was assembled and operated as a stand-alone detector for a period of approximately two years. In this report, the results from CUORE-0 and the current status and physics potential of CUORE are presented. 
Two neutrino double beta decay $(2 v)$ is an extremely rare nuclear transition from a nucleus (Z,A) to its isobar, $(Z+2, A)$, with the emission of two electrons and two electron antineutrinos. The transition occurs via a Standard Model allowed process and has been observed in several nuclides with half-lives of the order of $10^{19}-10^{24}$ years. Neutrinoless double-beta $(0 \mathrm{v})$ decay is a hypothesized process that, if observed, would establish the Majorana nature of the neutrino [1]. In the $0 v$ decay, two neutrons simultaneously decay into two protons, with two electrons and no neutrinos in the final state. The experimental signature of $0 v \beta \beta$ decay would be a peak in the summed electron energy spectrum at the $\mathrm{Q}_{\beta \beta}$ value for the isotope under study. The $0 v$ decay can occur through the exchange of a massive Majorana neutrino, and its occurrence violates lepton number conservation by two units, thus implying physics beyond the Standard Model.

\section{The CUORE detector}

The CUORE experiment will search for $0 \mathrm{v}$ decay of the isotope ${ }^{130} \mathrm{Te}$ using an array of $\mathrm{TeO}_{2}$ bolometers. The basic working principle of CUORE is based on the calorimetric technique: the energy released in an absorber is measured through its temperature rise, which is read out by a sensitive thermal sensor attached to the absorber. CUORE is made of 988 natural isotopic composition $\mathrm{TeO}_{2}$ detectors, mounted in a cylindrical compact structure of 19 towers, each made by 52 crystals arranged in 13 floors of 4 detectors each. The absorbers are cubic crystals $\left(5 \times 5 \times 5 \mathrm{~cm}^{3}\right.$ each), with a total active mass of $742 \mathrm{~kg}(206 \mathrm{~kg}$ ${ }^{130} \mathrm{Te}$ ). The temperature sensors that converts the thermal variation into an electrical signal are neutron transmutation doped (NTD) germanium thermistors, glued on the absorber. The crystals are arranged in a copper structure, which serves as the heat bath. When operated at cryogenic temperature $(\sim 10 \mathrm{mK})$, the heat capacity of a $750 \mathrm{~g} \mathrm{TeO}_{2}$ crystal is so low that an energy deposition of $1 \mathrm{MeV}$ produces a temperature rise of $\sim 100 \mu \mathrm{K}$. The corresponding variation of the NTD resistivity is of the order of few percent, and can be easily read out with low-noise room temperature electronics. The expected background rate in the Region of Interest (ROI) is 0.01 counts $/ \mathrm{keV} / \mathrm{kg} / \mathrm{y}$, mainly from contaminations from the surface and bulk of the bolometer components and from the shielding. To achieve this background level, stringent criteria were applied both on the selection of the materials used in the construction of the detector and in the cleaning procedure, handling, and storage of the materials. We worked very closely with the Shanghai Institute of Ceramics (SICCAS) to develop a procedure to grow extremely radio-pure $\mathrm{TeO}_{2}$ crystals [2]. Based on our measurements of cross sections for cosmogenic neutron activation of $\mathrm{TeO}_{2}$ [3], we also limited the amount of time these crystals were allowed to be above ground to be no more than 4 months. Moreover, the whole detector construction took place in a dedicated clean room (class 1000) in the CUORE hut, using a set of specially designed glove boxes, to keep the detector parts under a constant flow of nitrogen and out of contact with the rest of the environment of the clean room [4].

The CUORE hut is located at the LNGS underground facility at an average depth of $\sim 3600 \mathrm{~m}$ water equivalent. The muon flux at LNGS is $3 \times 10^{-8} / \mathrm{cm}^{2} / \mathrm{sec}$, about six orders of magnitude lower than that sea level [5]. A heavy shield consisting of layers of borated polyethylene, boric-acid powder, and lead bricks surrounds the cryostat to attenuate neutron and $\gamma$-ray backgrounds. More lead shielding is added inside the cryostat, including ancient Roman lead to further suppress the $\gamma$-rays from the cryostat materials. With a background index of 0.01 counts $/ \mathrm{keV} / \mathrm{kg} / \mathrm{y}$, the projected half-life sensitivity for CUORE is $9.5 \times 10^{25} \mathrm{y}(90 \%$ C.L.), corresponding to an upper limit on the effective Majorana mass in the range of 50-130 $\mathrm{meV}$, depending on the value of the nuclear matrix element chosen.

In order to host and cool down the CUORE detector, a custom made cryostat was built. The main challenge was to satisfy the cryogenic requirements and the low-radioactivity ones simultaneously. The CUORE cryostat is cryogen-free, and the first stage of cooling, down to $4 \mathrm{~K}$ is provided by 5 pulse tube coolers. The base temperature $<10 \mathrm{mK}$ is provided by a dilution refrigerator, designed and built by Leiden Cryogenics, with a cooling power of $3 \mu \mathrm{W}$ at $10 \mathrm{mK}$. The different stages of the cryostat with their thermal shields are made of OFHC (oxygen-free high thermal conductivity) copper, specially chosen for its low hydrogen content, low bulk radioactivity and high thermal conductivity. The detectors are surrounded by different lead shields. The first one is a 6-cm-thick lateral shield and a shield below the towers, made from ancient roman lead [6]. A $24 \mathrm{~cm}$ plate of modern low background lead (164 Bq/kg) shields the detector from above. An additional shield, $25 \mathrm{~cm}$ minimum thick, made out of modern lead $(15020 \mathrm{~Bq} / \mathrm{kg}$ ) surrounds the cryostat. A schematic drawing of the CUORE detector is shown in Figure 1 along with that of a typical human to set the scale on the size of the detector. Figure 2 shows the location of the CUORE experiment at the LNGS. The CUORE detector was assembled and installed at the LNGS over the last several years and is now fully complete. By the end of January, 2017, the detector had been cooled down to its operating temperature of $\sim 10 \mathrm{mK}$ and commissioning and data taking are planned to start in the near future. 

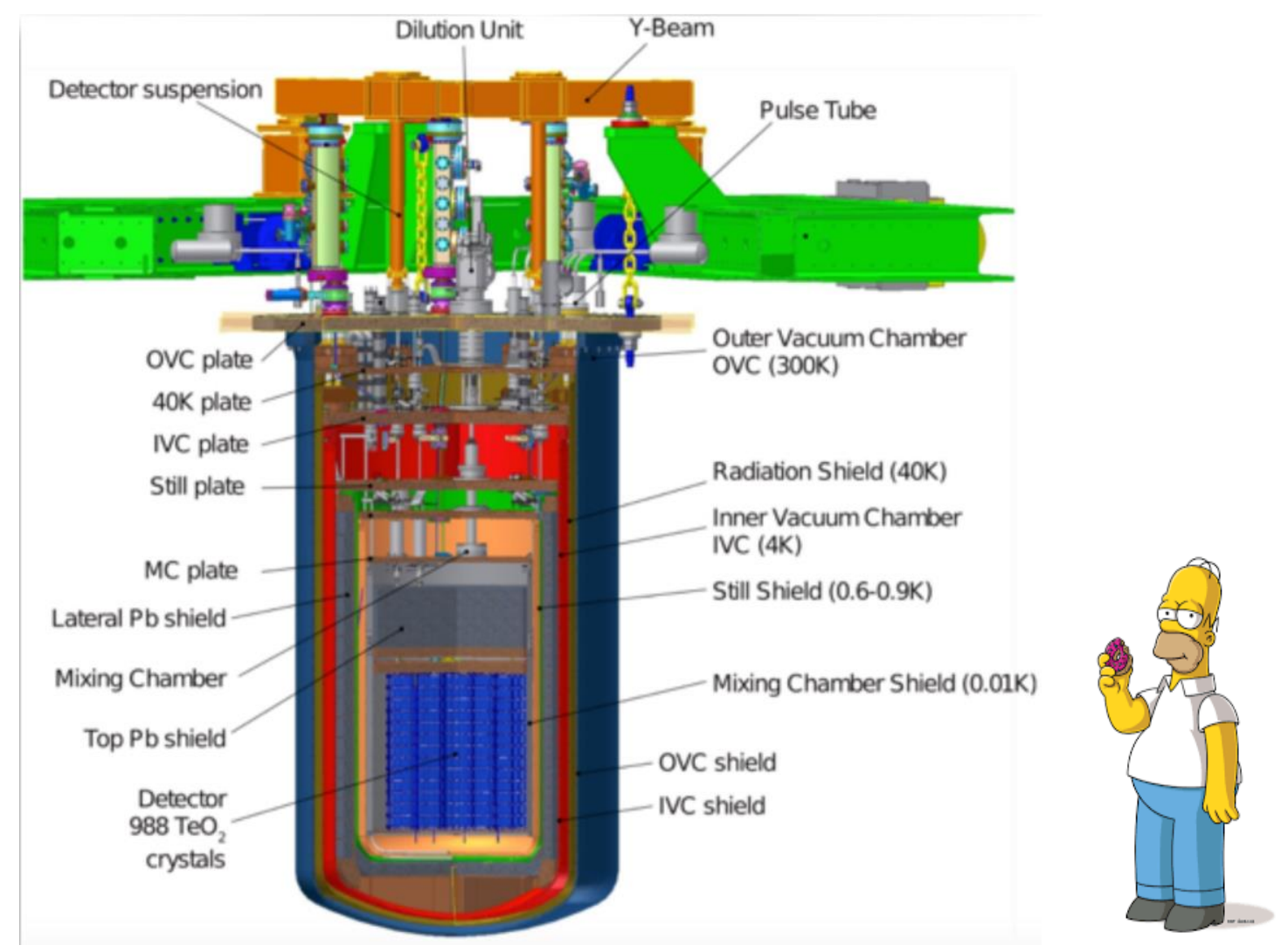

Figure 1. CUORE detector.

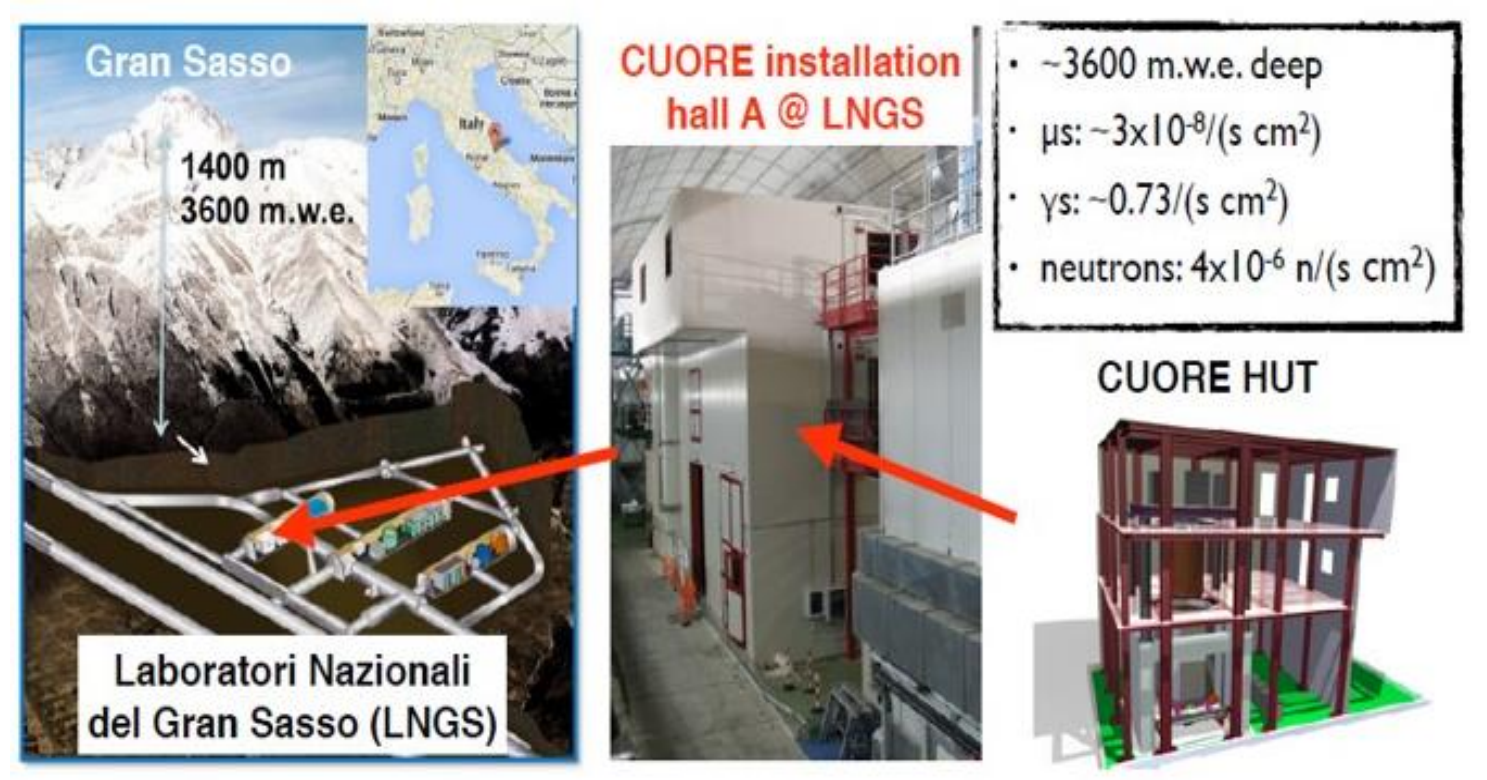

Figure 2. CUORE detector location at the Gran Sasso National Laboratory in Italy.

\section{The CUORE- 0 detector}

CUORE-0 was a single CUORE-like tower built using the low-background assembly techniques developed for CUORE. CUORE-0 was operated in the Hall A of LNGS from 2013 to 2015, using the old setup already used for the previous CUORE prototypes. Details on the CUORE-0 detector performance and analysis can be found in [7] and [8] while the CUORE-0 result on the search for $0 v \beta \beta$ decay is published in 
[9]. The detectors were calibrated monthly by inserting a source of thoriated wires close to the outer vessel of the cryostat. The data acquired between two consecutive calibration measurements are referred to as dataset. Using the known energy of gamma lines between $511 \mathrm{keV}$ and $2615 \mathrm{keV}$ we were able to evaluate the calibration curve for each bolometer and for each dataset. We used the strongest line in calibration data (2615 $\mathrm{keV}$ from ${ }^{208} \mathrm{Tl}$ ) to evaluate the detector response to a monoenergetic deposit near the ROI, for each bolometer and dataset. The physics-exposure-weighted mean of the resolution values for all of the bolometers is $4.9 \mathrm{keV}$ (FWHM), with a corresponding RMS of $2.9 \mathrm{keV}$. This demonstrates that the CUORE goal of $5 \mathrm{keV}$ energy resolution is feasible. We evaluated the background in the alpha-dominated region (between $2700 \mathrm{keV}$ and $3900 \mathrm{keV}$ ) as $0.016 \pm 0.001$ counts $/ \mathrm{keV} / \mathrm{kg} / \mathrm{y}, 6.8$ times less than the background achieved by Cuoricino in the same region, $0.110 \pm 0.001$ counts $/ \mathrm{keV} / \mathrm{kg} / \mathrm{y}$. This is proof that the background mitigation techniques adopted for the cleaning and assembly of the detector were effective. We searched for 0v decay in the CUORE- 0 spectrum corresponding to a total $\mathrm{TeO}_{2}$ exposure of $35.2 \mathrm{~kg}-\mathrm{y}\left(9.8 \mathrm{~kg}-\mathrm{y}\right.$ of $\left.{ }^{130} \mathrm{Te}\right)$. We performed a search in the interval $2470-2570 \mathrm{keV}$, using a function composed of three parameters: a signal peak at the $\mathrm{Q}_{\beta \beta}$-value of the transition, a peak at $2507 \mathrm{keV}$ from ${ }^{60} \mathrm{Co}$ gamma-ray summing, and a smooth continuum background attributed to multi-scatter Compton events from ${ }^{208} \mathrm{Tl}$ and surface alpha decays. The result of the search is shown in Fig. 3. We do not observe a peak at the expected $\mathrm{Q}_{\beta \beta}$ energy of $2527 \mathrm{keV}$. The background in the ROI is $0.058 \pm 0.004$ (stat) \pm 0.002 (syst) counts $/ \mathrm{keV} / \mathrm{kg} / \mathrm{y}$. With these data, we set a $90 \%$ C.L. lower bound on the $0 v$ decay half-life of $2.7 \times 10^{24} \mathrm{yr}$. Combining this result with the $19.75 \mathrm{~kg}$-y exposure of ${ }^{130} \mathrm{Te}$ from the Cuoricino experiment, we obtain a limit of $\mathrm{t}_{1 / 2}>4.0 \times 10^{24} \mathrm{yr}(90 \%$ C.L.), which is the most stringent limit to date on the ${ }^{130} \mathrm{Te} 0 v$ half-life [9].

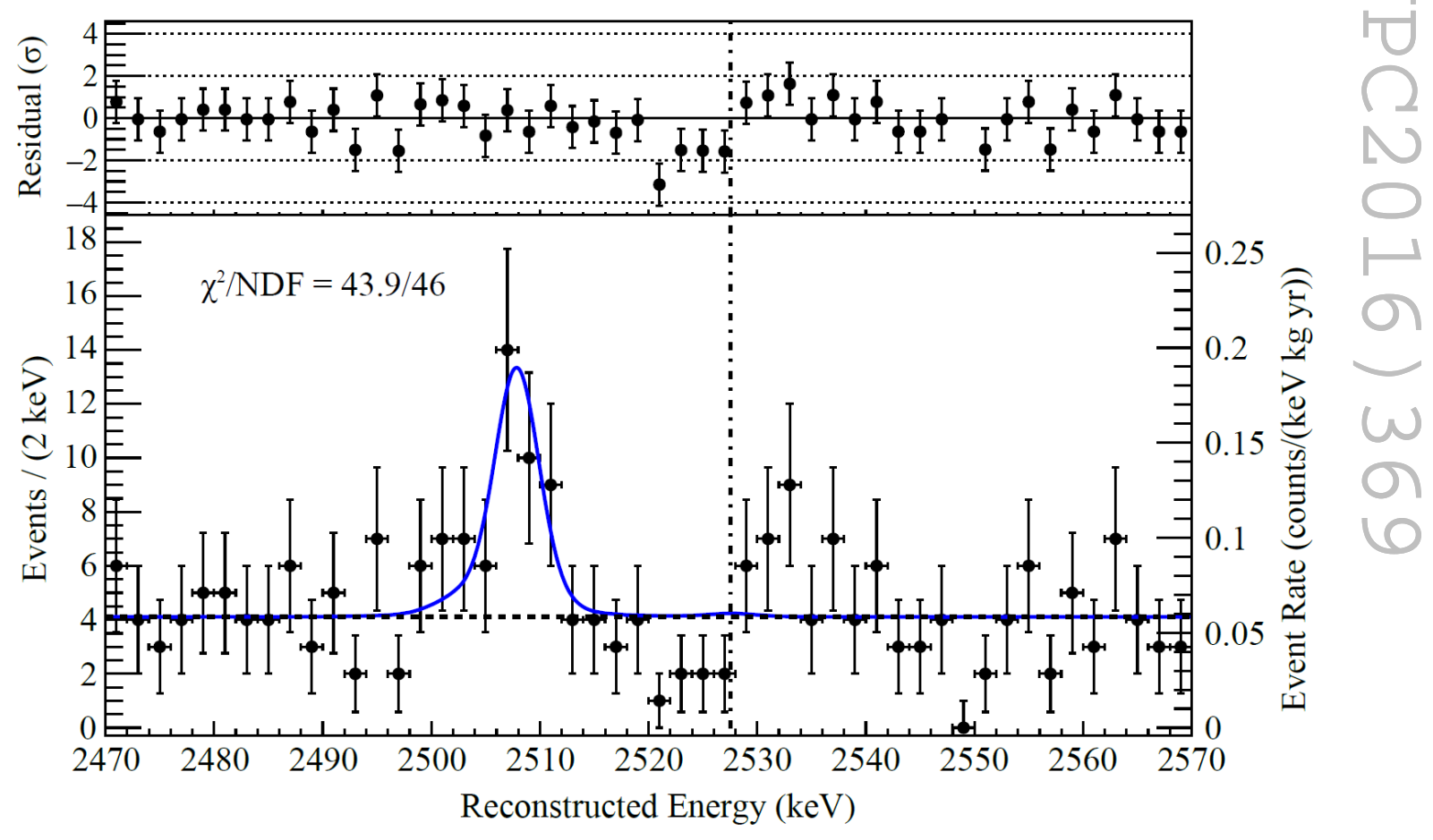

Figure 3. Portion of the CUORE-0 energy spectrum around the $\mathrm{Q}_{\beta \beta}$ endpoint energy of ${ }^{130} \mathrm{Te}$.

We also used the CUORE- 0 data for an extended and comprehensive study of the background sources that contribute to the count rate in the region of interest. We built a Geant4-based Monte Carlo (MC) simulation to study the contribution of the particle interaction in the entire CUORE-0 setup (including the detector components, the cryostat and the shielding). We used, as input to the simulation, the values of the material contaminations obtained from screening measurements (i.e. HPGe and NAA measurements) and we built the energy spectra obtained by simulating the contamination sources in different position of the setup. We simulated 57 sources, with activities obtained from of the CUORE-0 background data. In order to properly fit the data, the energy spectrum of the summed electrons from $2 v \beta \beta$ decay must be included. We found that the $2 v \beta \beta$ decay of ${ }^{130} \mathrm{Te}$ accounts for approximately $10 \%$ of the events in the region from $118 \mathrm{keV}$ to $2.5 \mathrm{MeV}$. The half-life value obtained for $2 v \beta \beta$ decay of ${ }^{130} \mathrm{Te}$ is $[8.2 \pm 0.2$ (stat.) \pm 0.6 (syst.) $]$ x $10^{20 \mathrm{y}}$. This result is the most precise measurement of the half-life of ${ }^{130} \mathrm{Te}$ to date. For more details, see [10]. 
Based on the experimental results from CUORE-0 and our extensive material screening measurements, we have also constructed an estimate of the expected background in the region of interest for the full CUORE detector. The results of this study are shown in Figure 4. It appears that our goal of 0.01 counts $/ \mathrm{keV} / \mathrm{kg} / \mathrm{yr}$ is within reach.

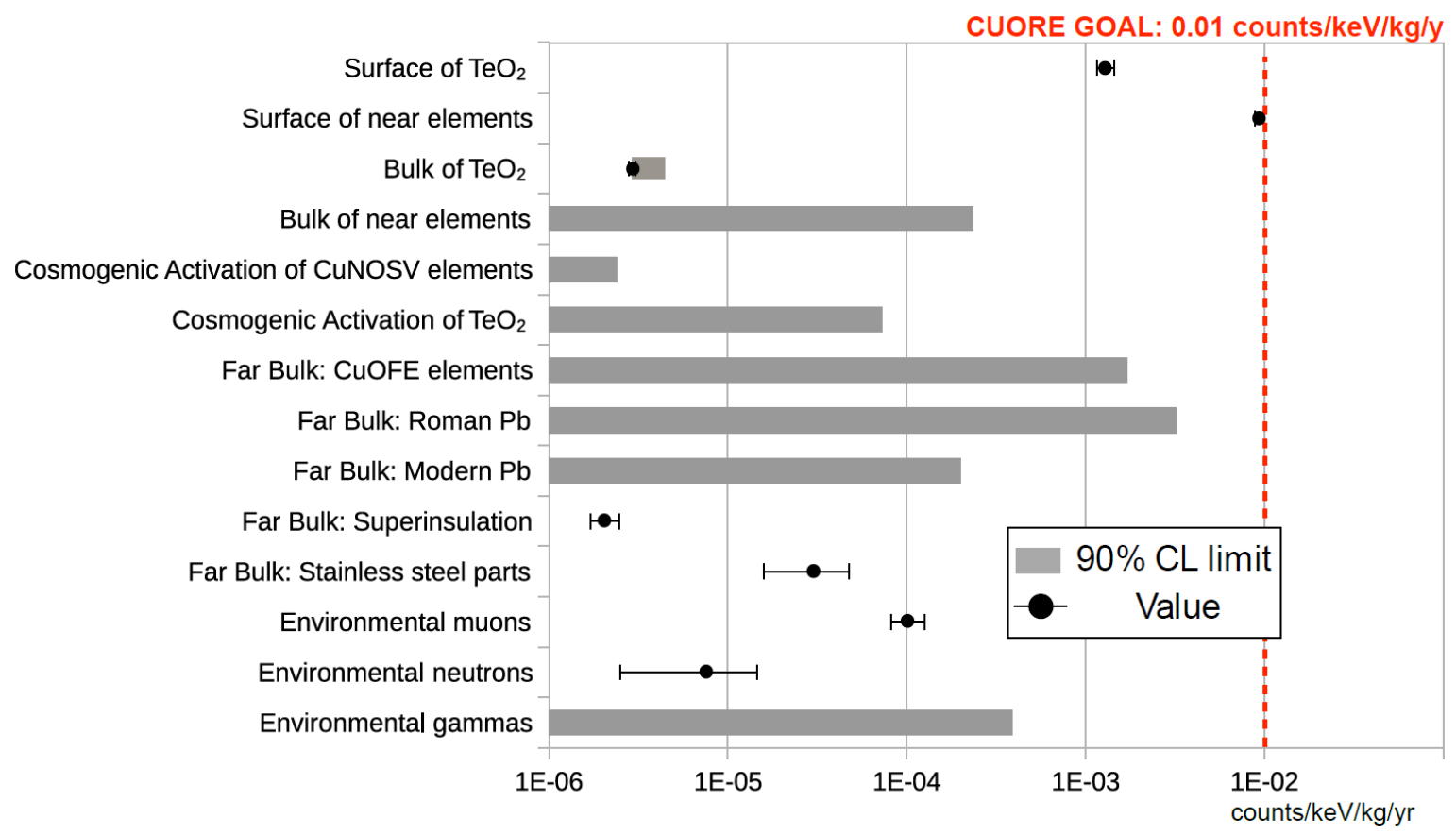

Figure 4. CUORE background buget.

From this, we can then estimate the potential sensitivity of CUORE for $0 v \beta \beta$ decay. These results are illustrated in Figure 5. From a 5-year data taking run, CUORE will have sensitivity at the 90\% confidence level to a neutrinoless double beta decay half-life of ${ }^{130} \mathrm{Te}$ of $9.5 \times 10^{25}$ years and to effective Majorana neutrino masses of 50-130 meV depending on the value of the nuclear matrix element.

\section{Future plans}

The bolometric technique has been shown to be one of the most promising technologies for future programs to explore the inverted mass hierarchy region. Recently, the CUPID (CUORE Upgrade with Particle IDentication) group of interest has formed, in order to coordinate R\&D efforts towards the goal of increasing the sensitivity to neutrinoless double beta decay. Different approaches are presently investigated, using different isotopes, additional sensors to detect scintillation or Cherenkov light, as well as exploring the viability of enriching ${ }^{130} \mathrm{Te}$. Strong R\&Ds efforts are currently being made to investigate a variety of techniques. The goal of CUPID is to probe the entire inverted hierarchy region, with a sensitivity on $\mathrm{t}_{1 / 2}$ of [2 - 5] x $10^{27}$ years with 10 years of data, with a corresponding limit on the effective Majorana neutrino mass of between 6 and $20 \mathrm{meV}$.

\section{Acknowledgements}

The CUORE Collaboration thanks the directors and staff of the Laboratori Nazionali del Gran Sasso and the technical staff of our laboratories. This work was supported by the Istituto Nazionale di Fisica Nucleare (INFN), the National Science Foundation, the Alfred P. Sloan Foundation, the University of Wisconsin Foundation, and Yale University. This material is also based upon work supported by the US Department of Energy (DOE) Office of Science, Office of Nuclear Physics. This research used resources of the National Energy Research Scientific Computing Center (NERSC). 


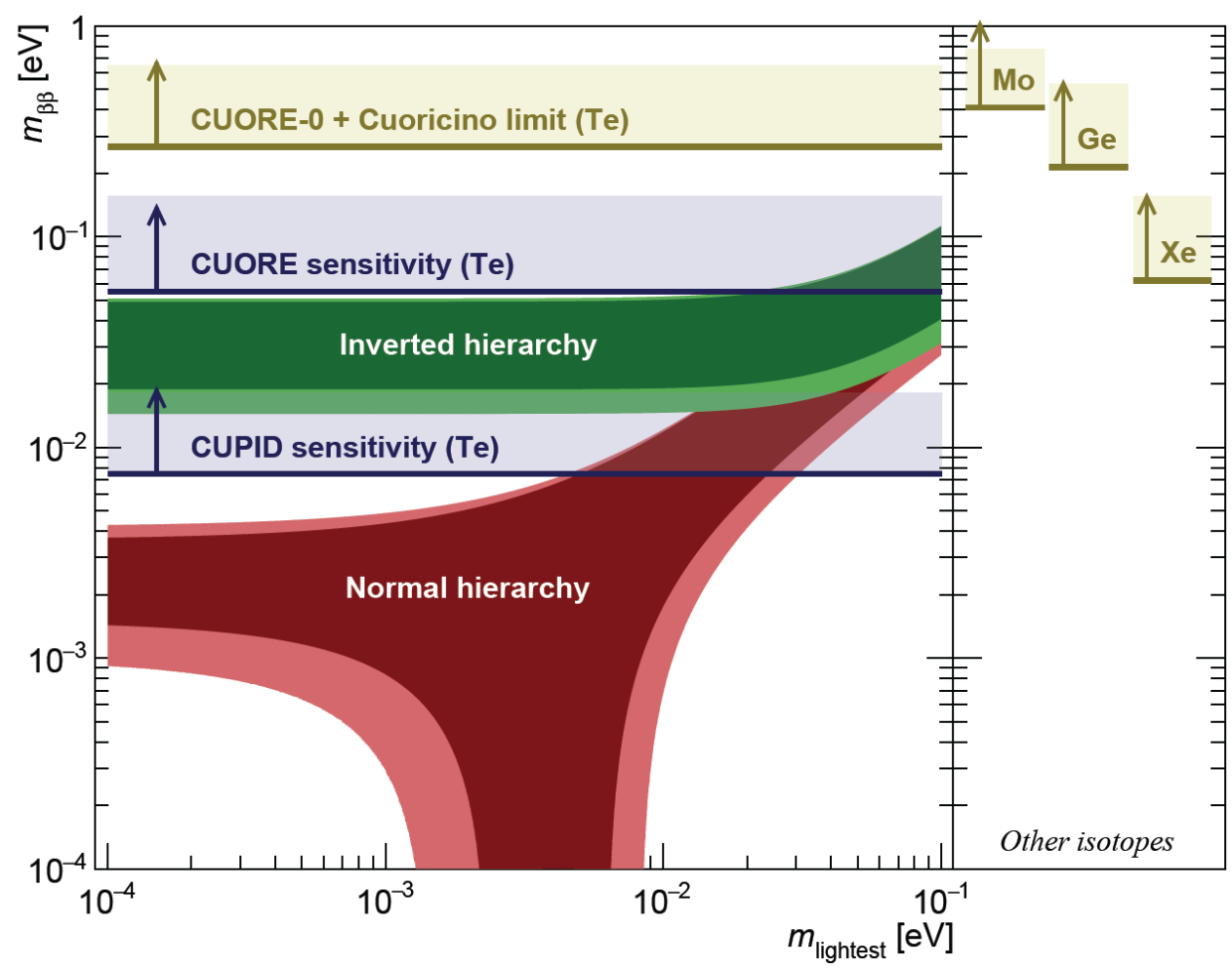

Figure 5. Estimated CUORE sensitivity.

\section{References}

[1] W. H. Furry, Phys. Rev. 56, 1184 (1939).

[2] C. Arnaboldi et al., J. Crystal Growth 312, 2999 (2010).

[3] B. S. Wang et al., Phys. Rev C 92, 024620 (2015).

[4] E. Buccheri et al., Nucl. Instrum. Meth. A 768, 130 (2014).

[5] M. Ambrosio et al., Phys. Rev. D 52, 3793, 1995.

[6] A. Alessandrello et al., Nucl. Instrum. Meth. B 142, 163 (1998).

[7] C. Alduino et al., JINST 11 P07009 (2016).

[8] C. Alduino et al., Phys. Rev. C 93, 045503 (2016).

[9] K. Alfonso et al., Phys. Rev. Lett. 115, 102502 (2015).

[10] C. Alduino et al., Eur. Phys. J. C. 77:13 (2017). 